\title{
A LOCAL EXISTENCE THEOREM \\ FOR A CLASS OF DELAY DIFFERENTIAL EQUATIONS
}

\author{
IOAN I. VRABIE
}

\begin{abstract}
The goal of this paper is to show that some classes of partial differential functional equations admit a natural formulation as ordinary functional differential equations in infinite dimensional Banach spaces. Moreover, the equations thus obtained are driven by continuous right-hand sides satisfying the compactness assumptions required by the infinite-dimensional version of a Peano-like existence theorem. Two applications, one to a semilinear wave equation with delay and another one to a pseudoparabolic PDE in Mechanics, are included.
\end{abstract}

\section{Introduction}

Let $X$ be a Banach space, $\tau \geq 0, X:=C([-\tau, 0] ; X), \sigma \in \mathbb{R}, f, g:[\sigma,+\infty) \times$ $X \rightarrow X$ be two continuous functions, and $\psi \in X$. If $u \in C([-\tau,+\infty) ; X)$ and $t \geq 0, u_{t}$ is defined by $u_{t}(s)=u(t+s)$ for each $s \in[-\tau, 0]$.

2010 Mathematics Subject Classification. Primary: 34K30, 35K70; Secondary: 35L71, 47H10, 47H30.

Key words and phrases. Delay differential equations; local existence; metric fixed point arguments; topological fixed point arguments; semilinear wave equation; pseudoparabolic equation.

This work was supported by a grant of the Romanian National Authority for Scientific Research, CNCS-UEFISCDI, project number PN-II-ID-PCE-2011-3-0052.

The author expresses his warmest thanks to the referees for their useful remarks and comments on the initial form of the manuscript. 
We consider the nonlinear delay differential evolution Cauchy problem

$$
\begin{cases}u^{\prime}(t)=f\left(t, u_{t}\right)+g\left(t, u_{t}\right) & \text { if } t \in[\sigma, T], \\ u(t)=\psi(t-\sigma) & \text { if } t \in[\sigma-\tau, \sigma],\end{cases}
$$

and we prove that, if $f$ is continuous and compact and $g$ is continuous and locally Lipschitz with respect to its second variable, problem (1.1) has at least one local classical solution. It should be noticed that all existence results which will follow can be completed by global existence results under the additional hypothesis that both $f$ and $g$ have sublinear growth. However, we are not going to touch upon here this kind of problems whose proofs, although based on a recent delay-type Gronwall Inequality established by Burlică and Roşu [4], are standard. For the basic results on delay differential equations the interested reader is referred to Driver [6], Halanay [9] and Hale [10].

A non-delayed version (1.1) was considered by Frigon and O'Reagan [8] who obtained a local existence result by using the Krasnosel'skiu Fixed Point Theorem [11]. For other existence results concerning delay equations and inclusions see Mitidieri and Vrabie [12] and [13].

We includ two applications of our abstract existence result. First, we show that, surprisingly, a second order semilinear delay wave equation can be rewritten under the form (1.1), where $f$ is continuous and compact and $g \equiv 0$ and so the local existence follows by means of usual finite-dimensional-type ODE's techniques. Here we exploit an idea of the author which has shown to be effective in the non-delayed case. See Vrabie [15, Theorem 10.4.1, p. 243]. Second, we prove that a pseudoparabolic problem arising in Mechanics can be transformed in the form (1.1) with $f$ and $g$ satisfying all the conditions mentioned before. As far as the second application is concerned, it should be noticed that a similar non-delayed problem was considered by Brill [3] who proved how to reduce it to an ordinary differential equation in an infinite-dimensional Banach space.

Definition 1.1. (a) A function $f:[\sigma,+\infty) \times X \rightarrow X$ is called compact if it maps bounded subsets in $[\sigma,+\infty) \times \mathcal{X}$ into relatively compact subsets in $X$.

(b) A function $g:[\sigma,+\infty) \times X \rightarrow X$ is called locally Lipschitz with respect to its last argument if for each $\psi \in \mathcal{X}$ and each $T>\sigma$ there exist $r=r(T, \psi)>0$ and $L=L_{T, \psi}>0$ such that

$$
\|g(t, u)-g(t, v)\| \leq L\|u-v\|, \quad \text { for each }(t, u),(t, v) \in[\sigma, T] \times D_{X}(\psi, r),
$$

where $D_{X}(\psi, r)$ denotes the closed ball of center $\psi$ and radius $r$ in $X$.

Definition 1.2. By a classical solution of problem (1.1) on $[\sigma-\tau, T]$ we mean a function $u:[\sigma-\tau, T] \rightarrow X$ which is of class $C^{1}$ on $[\sigma, T]$, satisfies $u^{\prime}(t)=f\left(t, u_{t}\right)+g\left(t, u_{t}\right)$ for each $t \in[\sigma, T]$ and $u(t)=\psi(t-\sigma)$ for each $t \in$ $[\sigma-\tau, \sigma]$. By a classical solution of problem (1.1) on $[\sigma,+\infty)$ we mean a function 
$u:[\sigma-\tau,+\infty) \rightarrow X$ which is a classical solution of (1.1) on $[\sigma-\tau, T]$ for each $T>\sigma$.

For easy reference, we recall next some results we will use in that follows. From the Mazur Theorem, see e.g. Vrabie [15, Theorem A.1.3, p. 292], we get:

Lemma 1.3. Let $K$ be a compact subset in $X$ and let $\mathcal{F}$ a family of continuous functions from $[a, b]$ to $K$. Then, for each $t \in[a, b]$, the set

is relatively compact in $X$.

$$
\left\{\int_{a}^{t} f(s) d s ; f \in \mathcal{F}\right\}
$$

A more general compactness result due to Becker [2] can be found in Cârjă, Necula and Vrabie [5, Lemma 1.5.1, p. 14].

Theorem 1.4 (Schauder). Let $X$ be a Banach space. If $K \subseteq X$ is nonempty, closed and convex, the mapping $\mathcal{M}: K \rightarrow K$ is continuous and $\mathcal{M}(K)$ is relatively compact, then $\mathcal{M}$ has at least one fixed point, i.e. there exists $\xi \in K$ such that $\mathcal{M}(\xi)=\xi$.

See Schauder [14] or Dunford and Schwartz [7, Theorem 5, p. 456].

Theorem 1.5 (Arzełà-Ascoli). Let $X$ be a Banach space. A subset $\mathcal{F}$ in $C([a, b] ; X)$ is relatively compact if and only if:

(a) $\mathcal{F}$ is equicontinuous on $[a, b]$;

(b) there exists a dense subset $D$ in $[a, b]$ such that, for each $t \in D$,

$$
\mathcal{F}(t)=\{f(t) ; f \in \mathcal{F}\}
$$

is relatively compact in $X$.

See Vrabie [15, Theorem A.2.1, p. 296].

Theorem 1.6 (Sobolev, Rellich, Kondrašov). Let $\Omega$ be a nonempty, open and bounded subset in $\mathbb{R}^{d}, d \geq 1$, whose boundary $\Sigma$ is of class $C^{1}$. Let $m \in \mathbb{N}$ and $p, q \in[1,+\infty)$.

(a) If $m p<d$ and $q<d p /(d-m p)$, then $W^{m, p}(\Omega)$ is compactly imbedded in $L^{q}(\Omega)$.

(b) If $m p=d$ and $q \in[1,+\infty)$, then $W^{m, p}(\Omega)$ is compactly imbedded in $L^{q}(\Omega)$.

(c) If $m p>d$, then $W^{m, p}(\Omega)$ is compactly imbedded in $C(\bar{\Omega})$.

See Adams [1, Theorem 5.4, p. 97 and Theorem 6.2, p. 144].

Definition 1.7. Let $h: \mathbb{R}_{+} \times \bar{\Omega} \times \mathbb{R} \rightarrow \mathbb{R}$ be a given arbitrary function. The operator $h_{p}: \mathbb{R}_{+} \times D_{p} \subset \mathbb{R}_{+} \times L^{p}(\Omega) \rightarrow L^{1}(\Omega)$ be defined by

$$
h_{p}(t, u)(x)=h(t, x, u(x)), \quad \text { for each } t \in \mathbb{R}_{+}, u \in D_{p} \text { and a.e. } x \in \Omega,
$$


where $D_{p}=\left\{u \in L^{p}(\Omega) ; h(t, \cdot, u(\cdot)) \in L^{1}(\Omega)\right.$ for each $\left.t \in \mathbb{R}_{+}\right\}$is called the superposition operator on $L^{p}(\Omega)$ associated to the function $h$.

We say that the superposition operator on $L^{p}(\Omega)$ associated to $h$ is welldefined on $L^{p}(\Omega)$, if $D_{p}=L^{p}(\Omega)$ and $h_{p}$ maps $\mathbb{R}_{+} \times D_{p}$ in $L^{p}(\Omega)$. We say that $h_{p}$ is well-defined on $C(\bar{\Omega})$, if $C(\bar{\Omega}) \subset D_{p}$ and $h_{p}$ maps $\mathbb{R}_{+} \times C(\bar{\Omega})$ in $C(\bar{\Omega})$.

Lemma 1.8. Assume that $h: \mathbb{R}_{+} \times \bar{\Omega} \times \mathbb{R} \rightarrow \mathbb{R}$ satisfies:

$\left(\mathrm{h}_{1}\right)$ for each $(t, x) \in \mathbb{R}_{+} \times \bar{\Omega}, u \mapsto h(t, x, u)$ is continuous;

$\left(\mathrm{h}_{2}\right)$ for each $u \in \mathbb{R},(t, x) \mapsto h(t, x, u)$ is measurable;

$\left(\mathrm{h}_{3}\right)$ for each $T>0$ and $r>0$, the restriction of $h$ to $[0, T] \times \bar{\Omega} \times[-r, r]$ is bounded.

Let $p \in[1,+\infty]$ and $h_{p}: \mathbb{R}_{+} \times D_{p} \subset \mathbb{R}_{+} \times L^{p}(\Omega) \rightarrow L^{1}(\Omega)$ be the superposition operator on $L^{p}(\Omega)$ associated to $h$. Then

(a) $L^{\infty}(\Omega) \subset D_{p}$. If $p=+\infty, D_{\infty}=L^{\infty}(\Omega)$ and $h_{\infty}$ is well-defined on $L^{\infty}(\Omega)$. In addition, for each $t \in \mathbb{R}_{+}$, the function $u \mapsto h_{\infty}(t, u)$ is continuous from $L^{\infty}(\Omega)$ into itself and, for each $u \in L^{\infty}(\Omega)$, the function $t \mapsto h_{\infty}(t, u)$ is strongly measurable $\left({ }^{1}\right)$. Also in this case, i.e. $p=$ $+\infty$, if $h$ is jointly continuous, then $h_{\infty}$ is well-defined on $C(\bar{\Omega})$ and continuous from $\mathbb{R}_{+} \times C(\bar{\Omega})$ to $C(\bar{\Omega})$.

(b) If $p \in[1,+\infty)$ and for each $T>0$ there exist $a_{T}>0$ and $b_{T} \in \mathbb{R}$ such that

$$
|h(t, x, u)| \leq a_{T}|u|^{p}+b_{T},
$$

for each $(t, x, u) \in[0, T] \times \bar{\Omega} \times \mathbb{R}$, then $D_{p}=L^{p}(\Omega)$, and, for each $t \in \mathbb{R}_{+}$, the function $u \mapsto h_{p}(t, u)$ is continuous from $L^{p}(\Omega)$ to $L^{1}(\Omega)$. In addition, for each $u \in L^{p}(\Omega)$, the function $t \mapsto h_{p}(t, u)$ is strongly measurable.

(c) If $p=1$ and $h$ satisfies (1.2) then, for each $r \in[1,+\infty]$, the restriction of $h_{1}$ to $\mathbb{R}_{+} \times L^{r}(\Omega)$ coincides with $h_{r}$ which is well-defined on $L^{r}(\Omega)$. In addition, for each $t \in \mathbb{R}_{+}$, the function $u \mapsto h_{1}(t, u)$ is continuous from $L^{r}(\Omega)$ into itself and, for each $u \in L^{r}(\Omega)$, the function $t \mapsto h_{1}(t, u)$ is strongly measurable from $\mathbb{R}_{+}$to $L^{r}(\Omega)$. Finally, for each $T>0$, we have

$$
\left\|h_{1}(t, u)\right\|_{L^{r}(\Omega)} \leq a_{T}\|u\|_{L^{r}(\Omega)}+\left|b_{T}\right| \mu(\Omega)^{1 / r}
$$

for each $t \in[0, T]$, and $u \in L^{r}(\Omega)$, where $a_{T}$ and $b_{T}$ are given by $(\mathrm{b})$, and $\mu(\Omega)$ is the Lebesgue measure of $\Omega$.

For the proof see Vrabie [15, Lemma A.6.1, p. 313].

$\left({ }^{1}\right)$ See Vrabie [15, Definition 1.1.1 (iii), p. 2]. 


\section{The main abstract existence result}

The goal of this section is to prove a local existence result referring to the Cauchy problem (1.1).

TheOREM 2.1. Let $f:[\sigma,+\infty) \times X \rightarrow X$ be continuous and compact, and let $g:[\sigma,+\infty) \times X \rightarrow X$ be continuous on $[\sigma,+\infty) \times \mathcal{X}$ and locally Lipschitz with respect to its last argument. Then, for each $\psi \in \mathcal{X}$, there exists $T>\sigma$ such that (1.1) has at least one classical solution, in the sense of Definition 1.2, defined on $[\sigma, T]$. If, in addition, there exist $k_{1}>0$ and $k_{2}>0$ such that

$$
\|f(t, v)\|+\|g(t, v)\| \leq k_{1}\|v\|_{x}+k_{2}, \quad \text { for each }(t, v) \in[\sigma,+\infty) \times X
$$

then each classical solution of (1.1) can be continued up to a global one, i.e. defined on $[\sigma-\tau,+\infty)$.

We shall prove this theorem with the help of two lemmas below which extend from some previous results referring to the non-delayed case, established in Vrabie [15, Lemma 10.2.2, p. 233 and Lemma 10.3.4, p. 234]. It should be noticed however that the proofs of both lemmas below are different from the proof of their just mentioned counterparts.

Lemma 2.2. Let $g: \mathbb{R} \times X \rightarrow X$ be continuous on $\mathbb{R} \times X$ and Lipschitz on $X$ with Lipschitz constant $\ell>0$, i.e.

$$
\|g(t, u)-g(t, v)\| \leq \ell\|u-v\|_{x}, \quad \text { for each } t \in \mathbb{R} \text { and each } u, v \in X .
$$

Then, for each $(\sigma, \psi) \in \mathbb{R}_{+} \times X$, each $T>\sigma$ with $(T-\sigma) \ell<1$ and each $h \in L^{1}(\sigma, T ; X)$, the problem

$$
\begin{cases}u^{\prime}=g\left(t, u_{t}\right)+h(t) & \text { if } t \in[\sigma, T], \\ u(t)=\psi(t) & \text { if } t \in[\sigma-\tau, \sigma],\end{cases}
$$

has a unique solution defined on $[\sigma, T]$. In addition, for each $(\sigma, \psi)$ and $T$, fixed as above, the mapping $h \mapsto \mathcal{S}(h)$, i.e. the unique solution of problem (2.1) corresponding to $h$, satisfies

$$
\left\|\mathcal{S}\left(h_{1}\right)-\mathcal{S}\left(h_{2}\right)\right\|_{C([\sigma, T] ; X)} \leq \frac{1}{1-(T-\sigma) \ell}\left\|H_{1}-H_{2}\right\|_{C([\sigma, T] ; X)},
$$

where

$$
H_{i}(t)=\int_{\sigma}^{t} h_{i}(s) d s, \quad \text { for } i=1,2 \text { and } t \in[\sigma, T] .
$$

Proof. Let $\psi \in X, T>\sigma$ and $r>0$ be arbitrary but fixed and let us define

$$
C_{\psi}:=\{v \in C([\sigma-\tau, T] ; X) ; v(t)=\psi(t-\sigma), t \in[\sigma-\tau, \sigma]\} .
$$


Clearly $C_{\psi}$ is nonempty and closed in $C([\sigma-\tau, T] ; X)$ and thus, endowed with the natural distance on $C([\sigma-\tau, T] ; X)$, is a complete metric space. Let $h \in$ $L^{1}(\sigma, T ; X)$ and let us define $Q: C_{\psi} \rightarrow C([\sigma-\tau, T] ; X)$ by

$$
(Q u)(t)= \begin{cases}\psi(t-\sigma) & \text { if } t \in[\sigma-\tau, \sigma], \\ \xi+\int_{\sigma}^{t} g\left(s, u_{s}\right) d s+\int_{\sigma}^{t} h(s) d s & \text { if } t \in(\sigma, T],\end{cases}
$$

for each $u \in C_{\psi}$, where $\xi=\psi(0)$. Clearly $Q$ maps $C_{\psi}$ into itself. Moreover, for each $u, v \in C_{\psi}$ and $t \in[\sigma, T]$, we have

$$
\begin{aligned}
\|[Q(u)](t)-[Q(v)](t)\| & \leq \int_{\sigma}^{t}\left\|g\left(s, u_{s}\right)-g\left(s, v_{s}\right)\right\| d s \\
& \leq \int_{\sigma}^{t} \ell\left\|u_{s}-v_{s}\right\|_{X} d s \leq(T-\sigma) \ell\|u-v\|_{C([\sigma-\tau, T] ; X)},
\end{aligned}
$$

for each $u, v \in C_{\psi}$. As $(T-\sigma) \ell<1$, the inequality above shows that $Q$ is a strict contraction. Taking into account that $C_{\psi}$ is a complete metric space, by the Banach Fixed Point Theorem, it follows that $Q$ has a unique fixed point $u \in C_{\psi}$, which clearly is a classical solution of problem (2.1).

Let now $h_{1}$ and $h_{2}$ be two elements in $L^{1}(\sigma, T ; X)$ and let us observe that

$$
\begin{aligned}
& \left\|\left[\mathcal{S}\left(h_{1}\right)\right](t)-\left[\mathcal{S}\left(h_{2}\right)\right](t)\right\| \\
& \quad \leq\left\|\int_{\sigma}^{t}\left(h_{1}(s)-h_{2}(s)\right) d s\right\|+\int_{\sigma}^{t}\left\|g\left(s,\left(\mathcal{S}\left(h_{1}\right)\right)_{s}\right)-g\left(s,\left(\mathcal{S}\left(h_{2}\right)\right)_{s}\right)\right\| d s \\
& \quad \leq\left\|H_{1}-H_{2}\right\|_{C([\sigma, T] ; X)}+\ell \int_{\sigma}^{t}\left\|\left[\mathcal{S}\left(h_{1}\right)\right]_{s}-\left[\mathcal{S}\left(h_{2}\right)\right]_{s}\right\| d s,
\end{aligned}
$$

for each $t \in[\sigma, T]$. So,

$$
\begin{aligned}
\left\|\left[\mathcal{S}\left(h_{1}\right)\right](t)-\left[\mathcal{S}\left(h_{2}\right)\right](t)\right\| \leq \| H_{1}- & H_{2} \|_{C([\sigma, T] ; X)} \\
& +(T-\sigma) \ell\left\|\mathcal{S}\left(h_{1}\right)-\mathcal{S}\left(h_{2}\right)\right\|_{C([\sigma-\tau, T] ; X)},
\end{aligned}
$$

for each $t \in[\sigma, T]$. Taking into account the initial condition, we get

$$
\left\|\mathcal{S}\left(h_{1}\right)-\mathcal{S}\left(h_{2}\right)\right\|_{C([\sigma-\tau, T] ; X)}=\left\|\mathcal{S}\left(h_{1}\right)-\mathcal{S}\left(h_{2}\right)\right\|_{C([\sigma, T] ; X)} .
$$

Therefore

$$
\left\|\mathcal{S}\left(h_{1}\right)-\mathcal{S}\left(h_{2}\right)\right\|_{C([\sigma, T] ; X)} \leq \frac{1}{1-(T-\sigma) \ell}\left\|H_{1}-H_{2}\right\|_{C([\sigma, T] ; X)},
$$

for each $h_{1}, h_{2} \in L^{1}(\sigma, T ; X)$ and this completes the proof.

Lemma 2.3. Let $f: \mathbb{R} \times X \rightarrow X$ be a continuous function, with $f(\mathbb{R} \times X)$ relatively compact, and let $g: \mathbb{R} \times X \rightarrow X$ be continuous on $\mathbb{R} \times X$ and Lipschitz on $X$ with Lipschitz constant $\ell>0$, i.e.

$$
\|g(t, u)-g(t, v)\| \leq \ell\|u-v\|_{X}, \quad \text { for each } t \in \mathbb{R} \text { and each } u, v \in \mathcal{X} .
$$


Let us assume, in addition, that there exists $m>0$ such that

$$
\|g(t, 0)\| \leq m, \quad \text { for each } t \in \mathbb{R} .
$$

Then, for each $(\sigma, \psi) \in \mathbb{R} \times \mathcal{X}$ and each $T>\sigma$ with $(T-\sigma) \ell<1$, problem (1.1) has at least one solution defined on $[\sigma, T]$.

Proof. Let $r>0$ and $M>0$ be such that both

$$
\|f(t, v)\| \leq M
$$

for each $(t, v) \in \mathbb{R} \times \mathcal{X}$, and

$$
\begin{aligned}
\max \left\{\frac{(T-\sigma)(M+m)+\|\psi(\cdot-\sigma)\|_{C([\sigma-\tau, \sigma] ; X)}}{1-(T-\sigma) \ell}\right. & \\
\left.\|\psi(\cdot-\sigma)\|_{C([\sigma-\tau, \sigma] ; X)}\right\} & \leq r
\end{aligned}
$$

are satisfied. We emphasize that we can always find constants $r$ and $M$ enjoying the above properties. Indeed, since $f(\mathbb{R} \times \mathcal{X})$ is relatively compact, it is bounded and so, there exists $M>0$ satisfying (2.4). Next, let us fix $M>0$ as before and let us choose a sufficiently large $r>0$ satisfying (2.5). Again, this is also possible because, by hypothesis, $(T-\sigma) \ell<1$.

Now, let $\psi \in X$ be arbitrary but fixed, let $r>0$ and $T>\sigma$ as above, and let

$$
\begin{aligned}
C_{\psi}([\sigma-\tau, T] ; X) & :=\{v \in C([\sigma-\tau, T] ; X) ; v(t)=\psi(t-\sigma) \text { for } t \in[\sigma-\tau, \sigma]\}, \\
K & :=\left\{v \in C_{\psi}([\sigma-\tau, T] ; X) ;\|v(t)\| \leq r \text { for } t \in[\sigma, T]\right\} .
\end{aligned}
$$

Clearly $K$ is nonempty, bounded, closed and convex in $C([\sigma-\tau, \sigma] ; X)$. Let $v \in K$. In view of Lemma 2.2, it follows that the problem

$$
\begin{cases}u^{\prime}(t)=g\left(t, u_{t}\right)+f\left(t, v_{t}\right) & \text { if } t \in[\sigma, T], \\ u(t)=\psi(t) & \text { if } t \in[\sigma-\tau, \sigma),\end{cases}
$$

has a unique classical solution $u:=P(v)$. Clearly

$$
[P(v)](t)= \begin{cases}\psi(t-\sigma) & \text { if } t \in[\sigma-\tau, \sigma) \\ \psi(0)+\int_{\sigma}^{t} g\left(s,[P(v)]_{s}\right) d s+\int_{\sigma}^{t} f\left(s, v_{s}\right) d s & \text { if } t \in[\sigma, T] .\end{cases}
$$

We distinguish between two possible cases.

Case 1. There exists $t \in[\sigma-\tau, \sigma]$ such that

$$
\|[P(v)](t)\|=\|P(v)\|_{C([\sigma-\tau, T] ; X)},
$$

a case in which, due to the initial condition, we have

$$
\|P(v)\|_{C([\sigma-\tau, T] ; X)}=\|\psi(\cdot-\sigma)\|_{C([\sigma-\tau, \sigma] ; X)} \leq r .
$$


Case 2. There exists $t \in(\sigma, T]$ such that (2.6) holds true. Then, from (2.3) and (2.4), we get

$$
\begin{aligned}
& \|[P(v)](t)\| \leq\|\psi(\cdot-\sigma)\|_{C([\sigma-\tau, \sigma] ; X)} \\
& \quad+\int_{\sigma}^{t}\left(\left\|g\left(s,[P(v)]_{s}\right)-g(s, 0)\right\|+\|g(s, 0)\|\right) d s+\int_{\sigma}^{t}\left\|f\left(s, v_{s}\right)\right\| d s \\
& \leq\|\psi(\cdot-\sigma)\|_{C([\sigma-\tau, \sigma] ; X)}+(T-\sigma) \ell\|P(v)\|_{C([\sigma-\tau, T] ; X)}+(T-\sigma)(M+m) .
\end{aligned}
$$

A simple calculation involving (2.5) shows that

$$
\|P(v)\|_{C([\sigma-\tau, T] ; X)} \leq \frac{(T-\sigma)(M+m)+\|\psi(\cdot-\sigma)\|_{C([\sigma-\tau, \sigma] ; X)}}{1-(T-\sigma) \ell} \leq r,
$$

which shows that $P(K) \subseteq K$.

Next, let us define $F: K \rightarrow C([\sigma, T] ; X)$ by

$$
F(v)(t)=\int_{\sigma}^{t} f\left(s, v_{s}\right) d s, \quad \text { for each } v \in K \text { and each } t \in[\sigma, T] .
$$

As $f(\mathbb{R} \times \mathcal{X})$ is relatively compact, from Lemma 1.3 , it follows that, for each $t \in[\sigma, T]$, the set $\{F(v)(t) ; v \in K\}$ is relatively compact in $X$. Moreover, $\{F(v) ; v \in K\}$ is equicontinuous on $[\sigma, T]$. Indeed, from (2.3), we conclude that

$\|F(v)(t)-F(v)(s)\| \leq M|t-s|, \quad$ for each $v \in K$ and each $t, s \in[\sigma, T]$.

Thanks to Arzelà-Ascoli Theorem 1.5, the set $F(K)$ is relatively compact in $C([\sigma, T] ; X)$. At this point, let us observe that from $(2.2)$ in Lemma 2.2, we have

$$
\|P(v)-P(w)\|_{C([\sigma, T] ; X)} \leq \frac{1}{1-(T-\sigma) \ell}\|F(v)-F(w)\|_{C([\sigma, T] ; X)},
$$

for each $v, w \in K$. To prove that $P(K)$ is relatively compact, let $\left(v_{n}\right)_{n}$ be an arbitrary sequence in $K$. By the compactness arguments above, we may assume with no loss of generality that $\left(F\left(v_{n}\right)\right)_{n}$ is a Cauchy sequence in $C([\sigma, T] ; X)$. From (2.7) it follows that

$$
\left\|P\left(v_{n}\right)-P\left(v_{m}\right)\right\|_{C([\sigma, T] ; X)} \leq \frac{1}{1-(T-\sigma) \ell}\left\|F\left(v_{n}\right)-F\left(v_{m}\right)\right\|_{C([\sigma, T] ; X)},
$$

for each $n, m \in \mathbb{N}$. So, $\left(P\left(v_{n}\right)\right)_{n}$ is a Cauchy sequence in $C([\sigma, T] ; X)$. Since $P(v)(t)=\psi(t-\sigma)$ for each $t \in[\sigma-\tau, \sigma]$, this clearly implies that $\left(P\left(v_{n}\right)\right)_{n}$ is a Cauchy sequence also in $C([\sigma-\tau, T] ; X)$. Consequently, $P(K)$ is relatively compact. In addition, from (2.7), the continuity of $f$ and Lemma 2.2, it follows that $P$ is continuous from $K$ into itself. By Schauder Fixed Point Theorem 1.4, it follows that $P$ has at least one fixed point $u \in K$, which obviously is a classical solution of (1.1).

We can now proceed to the proof of Theorem 2.1. 
Proof. Let $(\sigma, \psi) \in \mathbb{R} \times X$. Since $f$ and $g$ are continuous, there exist $T>\sigma$, $r>0$ and $M>0$ such that

$$
\|f(t, v)\| \leq M \quad \text { and } \quad\|g(t, v)\| \leq M,
$$

for each $(t, v) \in[\sigma, T] \times D_{X}(\psi, r)$. Let us define $\rho: X \rightarrow X$ by

$$
\rho(v)= \begin{cases}v & \text { for } y \in D_{X}(\psi, r), \\ \frac{r}{\|v-\psi\|}(v-\psi)+\psi & \text { for } v \in \mathcal{X} \backslash D_{X}(\psi, r),\end{cases}
$$

and let us observe that $\rho$ maps $X$ into $D_{X}(\psi, r)$ and is Lipschitz continuous on $X$ with Lipschitz constant 2 . Next, let us define $f_{r}:[\sigma, T] \times X \rightarrow X$ by

$$
f_{r}(t, v)=f(t, \rho(v)), \quad \text { for each }(t, v) \in[\sigma, T] \times X,
$$

and $g_{r}:[\sigma, T] \times X \rightarrow X$ by $g_{r}(t, v)=g(t, \rho(v))$, for each $(t, v) \in[\sigma, T] \times X$. As $f$ is compact, $f\left([\sigma, T] \times D_{x}(\psi, r)\right)$ is relatively compact. Accordingly, $f_{r}$ is continuous and $f_{r}([\sigma, T] \times \mathcal{X})$ is relatively compact. Analogously, from the choice of $\sigma, T$ and $r$, we deduce that $g_{r}$ is continuous and bounded on $[\sigma, T] \times \mathcal{X}$, and Lipschitz continuous on $X$ with Lipschitz constant $2 \ell$. From Lemma 2.3, we know that the Cauchy problem

$$
\begin{cases}u^{\prime}(t)=f_{r}\left(t, u_{t}\right)+g_{r}\left(t, u_{t}\right) & \text { if } t \in[\sigma, T], \\ u(t)=\psi(t-\sigma) & \text { if } t \in[\sigma-\tau, \sigma],\end{cases}
$$

has at least one solution $u:[\sigma, T] \rightarrow X$. As $u$ is continuous at $t=\sigma$, it follows that $t \mapsto u_{t}$ is continuous at $t=\sigma$. As $u_{\sigma}=\psi$, diminishing $T>\sigma$ if necessary, we have $\left\|u_{t}-\psi\right\| \leq r$ for each $t \in[\sigma, T]$. But this means that we have both $f_{r}\left(t, u_{t}\right)=f\left(t, u_{t}\right)$ and $g_{r}\left(t, u_{t}\right)=g\left(t, u_{t}\right)$. Consequently, $u:[\sigma, T] \rightarrow X$ is a classical solution of problem (1.1) and this completes the proof.

\section{An application}

We state now a Peano-type local existence theorem referring to a specific form of (1.1), i.e. the nonlinear delay differential evolution Cauchy problem

$$
\begin{cases}u^{\prime}(t)=f\left(t, u_{t}\right) & \text { if } t \in[\sigma, T], \\ u(t)=\psi(t-\sigma) & \text { if } t \in[\sigma-\tau, \sigma] .\end{cases}
$$

The theorem below is a direct consequence of Theorem 2.1.

Theorem 3.1. If $f:[\sigma,+\infty) \times X \rightarrow X$ is continuous and compact, then for each $\psi \in X$ there exists $T=T(\psi)>\sigma$ such that the delay initial-value problem (3.1) has at least one classical solution $u:[\sigma-\tau, T] \rightarrow X$. If, in addition, there exist $k_{1}>0$ and $k_{2}>0$ such that

$$
\|f(t, v)\| \leq k_{1}\|v\|_{x}+k_{2}, \quad \text { for each }(t, v) \in[\sigma,+\infty) \times X,
$$


then each classical solution of (1.1) can be continued up to a global one, i.e. defined on $[\sigma-\tau,+\infty)$.

We will derive next an abstract result referring to delay initial-value problems governed by compact perturbations of infinitesimal generators of $C_{0}$-groups. So, let us consider the delay initial-value problem in the Banach space $X$

$$
\begin{cases}u^{\prime}(t)=A u(t)+F\left(t, \int_{-\tau}^{0} u(t+s) d s\right) & \text { if } t \in[0, T] \\ u(t)=\varphi(t) & \text { if } t \in[-\tau, 0] .\end{cases}
$$

Theorem 3.2. Let $A: D(A) \subseteq X \rightarrow X$ be the infinitesimal generator of a $C_{0}$-group of linear operators $\{G(t): X \rightarrow X ; t \in \mathbb{R}\}$ and let $F: \mathbb{R}_{+} \times X \rightarrow X$ be a continuous and compact function. Then, for each $\varphi \in X$, there exists $T=$ $T(\varphi)>0$ such that the delay evolution initial-value problem (3.2) has at least one mild solution defined on $[0, T]$. If, in addition, there exist $k_{1}>0$ and $k_{2}>0$ such that

$$
\|F(t, u)\| \leq k_{1}\|u\|+k_{2}, \quad \text { for each }(t, u) \in \mathbb{R}_{+} \times X,
$$

then each mild solution of (3.2) can be continued up to a global one, i.e. defined on $[-\tau,+\infty)$.

Proof. We will show that problem (3.2) can be transformed into an ordinary delay differential equation of the form

$$
\begin{cases}u^{\prime}(t)=f\left(t, u_{t}\right) & \text { if } t \in[0, T] \\ u(t)=\psi(t) & \text { if } t \in[-\tau, 0]\end{cases}
$$

where $f: \mathbb{R}_{+} \times X \rightarrow X$ is continuous and compact and then we will make use of the infinite-dimensional variant of Peano Local Existence Theorem 3.1. First, let us recall that $u:[-\tau, T] \rightarrow X$ is a mild solution of problem (3.2) if and only if $u$ satisfies

$$
u(t)= \begin{cases}\varphi(t) & \text { if } t \in[-\tau, 0), \\ G(t) \varphi(0)+\int_{0}^{t} G(t-s) F\left(s, \int_{-\tau}^{0} u(s+\theta) d \theta\right) d s & \text { if } t \in[0, T] .\end{cases}
$$

But $G(t)$ is invertible and so, we get

$$
\begin{aligned}
& G^{-1}(t) u(t)=G^{-1}(t) \varphi(t), \quad t \in[-\tau, 0), \\
& G^{-1}(t) u(t)=\varphi(0)+\int_{0}^{t} G^{-1}(s) F\left(s, \int_{-\tau}^{0} u(s+\theta) d \theta\right) d s,
\end{aligned}
$$

for $t \in[0, T]$. Let us denote $G^{-1}(t) u(t)$ by $U(t)$ for $t \in[-\tau, T]$. Then, the integral equation above takes the equivalent form

$$
U(t)=\varphi(0)+\int_{0}^{t} G^{-1}(s) F\left(s, \int_{-\tau}^{0} G(s+\theta) U(s+\theta) d \theta\right) d s,
$$


for $t \in[0, T]$. Now, let us define the function $f: \mathbb{R}_{+} \times X \rightarrow X$ by

$$
f(t, v):=G^{-1}(t) F\left(t, \int_{-\tau}^{0} G(t+\theta) v(\theta) d \theta\right)
$$

for each $(t, v) \in \mathbb{R}_{+} \times X$. So, $u$ is a mild solution of problem (3.2) if and only if $U$ is a classical solution of initial-value problem (3.3), where $f$ is defined as above and $\psi(t)=G^{-1}(t) \varphi(t)$ for each $t \in[-\tau, 0]$. Since $F$ is continuous and compact, it readily follows that $f$ enjoys the very same properties. So, (3.3) can be handled by Theorem 3.1 .

\section{A semilinear wave equation with delay}

Here we will analyze an example showing that Theorem 3.1 is very suitable in the study of second order semilinear hyperbolic problems on bounded domains in $\mathbb{R}^{d}, d \geq 1$. It is interesting to notice that this example proves that such kind of problems are, in some sense, of "finite-dimensional" nature.

So, let $\Omega$ be a nonempty bounded and open subset in $\mathbb{R}^{d}, d \geq 1$, with $C^{1}$ boundary $\Sigma$, let $Q_{+}=\mathbb{R}_{+} \times \Omega, Q_{\tau}=[-\tau, 0] \times \Omega, \Sigma_{+}=\mathbb{R}_{+} \times \Sigma$ and let us consider the initial-value problem for the damped wave equation with delay

$$
\begin{cases}\frac{\partial^{2} u}{\partial t^{2}}(t, x)=\Delta u(t, x)+h\left(t, \int_{-\tau}^{0} u(t+s, x) d s\right) & \text { in } Q_{+}, \\ u(t, x)=0 & \text { on } \Sigma_{+}, \\ u(t, x)=\psi_{1}(t)(x) & \text { in } Q_{\tau} \\ \frac{\partial u}{\partial t}(t, x)=\psi_{2}(t)(x) & \text { in } Q_{\tau} .\end{cases}
$$

Problem (4.1) represents a closed loop system of a control problem associated to wave propagation in $\Omega$ in the (more realistic) case in which the synthesis operator $h$ reacts after some delay $\tau$.

As usual, if $\mathbb{I}$ is an interval, $p \in[1,+\infty)$ and $u: \mathbb{I} \times \Omega \rightarrow \mathbb{R}$ is a function such that for each $t \in \mathbb{I}, u(t, \cdot) \in L^{p}(\Omega)$, we identify it with a function from $\mathbb{I}$ to $L^{p}(\Omega)$, denoted for simplicity again by $u$. By virtue of this identification, for $t \in \mathbb{I}$, we write $u(t)(x)$ instead of $u(t, x)$ for almost every $x \in \Omega$, or even $u(t)$.

LEMMA 4.1. Let $h: \mathbb{R}_{+} \times \mathbb{R} \rightarrow \mathbb{R}$ be a continuous function for which there exist $\tilde{\ell}>0$ and $\tilde{m} \geq 0$ such that

$$
|h(t, y)| \leq \tilde{\ell}|y|+\widetilde{m}, \quad \text { for all }(t, y) \in \mathbb{R}_{+} \times \mathbb{R} .
$$

Then the superposition operator $h_{2}$ associated to $h$ is well-defined on $\mathbb{R}_{+} \times L^{2}(\Omega)$ in the sense of Definition 1.7 , is continuous from $\mathbb{R}_{+} \times L^{2}(\Omega)$ to $L^{2}(\Omega)$ and continuous and compact from $\mathbb{R}_{+} \times H_{0}^{1}(\Omega)$ to $L^{2}(\Omega)$.

Proof. The fact that $h_{2}$ is well-defined on $\mathbb{R}_{+} \times L^{2}(\Omega)$ and is continuous from $\mathbb{R}_{+} \times L^{2}(\Omega)$ to $L^{2}(\Omega)$ follows from (c) in Lemma 1.8. The continuity from 
$\mathbb{R}_{+} \times H_{0}^{1}(\Omega)$ to $L^{2}(\Omega)$ follows from the continuity from $\mathbb{R}_{+} \times L^{2}(\Omega)$ to $L^{2}(\Omega)$ and the continuity of the embedding $H_{0}^{1}(\Omega) \subseteq L^{2}(\Omega)$. We will prove next that $h_{2}$ is compact from $\mathbb{R}_{+} \times H_{0}^{1}(\Omega)$ to $L^{2}(\Omega)$. So, let $\left(\left(t_{n}, u_{n}\right)\right)_{n}$ be an arbitrary bounded sequence in $\mathbb{R}_{+} \times H_{0}^{1}(\Omega)$. Clearly $\left\{t_{n} ; n \in \mathbb{N}\right\}$ is relatively compact in $\mathbb{R}_{+}$. By Theorem 1.6, we know that $H_{0}^{1}(\Omega)$ is compactly embedded in $L^{2}(\Omega)$ and so, the set $\left\{u_{n} ; n \in \mathbb{N}\right\}$ is relatively compact in $L^{2}(\Omega)$. Recalling that the operator $h_{2}$ is continuous from $\mathbb{R}_{+} \times H_{0}^{1}(\Omega)$ to $L^{2}(\Omega)$, it follows that the set $\left\{h_{2}\left(t_{n}, u_{n}\right) ; n \in \mathbb{N}\right\}$ is relatively compact in $L^{2}(\Omega)$. So the superposition operator $h_{2}$ is compact from $\mathbb{R}_{+} \times H_{0}^{1}(\Omega)$ to $L^{2}(\Omega)$ and this completes the proof.

REMARK 4.2. As we will see in the proof of Theorem 4.3 below, problem (4.1) rewrites as an abstract semilinear evolution equation of form (3.2) in the product space

$$
X=\left(\begin{array}{c}
H_{0}^{1}(\Omega) \\
\times \\
L^{2}(\Omega)
\end{array}\right),
$$

where $A$ is the infinitesimal generator of a $C_{0}$-group of isometries in $X$. So, in that follows, by a mild solution of (4.1) we mean a mild solution of the corresponding semilinear evolution equation in the product space $X$.

THEOREM 4.3. Let $\Omega$ be a given nonempty bounded and open subset in $\mathbb{R}^{d}$, $d \geq 1$, with $C^{1}$ boundary $\Sigma, \tau \geq 0, h: \mathbb{R}_{+} \times \mathbb{R} \rightarrow \mathbb{R}$ and $\psi_{1} \in C\left([-\tau, 0] ; H_{0}^{1}(\Omega)\right)$ and $\psi_{2} \in C\left([-\tau, 0] ; L^{2}(\Omega)\right)$. Let us assume that

$\left(\mathrm{h}_{1}\right) h$ is continuous and there exist $\tilde{\ell}>0, \widetilde{m} \geq 0$ such that

$$
|h(t, y)| \leq \widetilde{\ell}|y|+\widetilde{m}, \quad \text { for all }(t, y) \in \mathbb{R}_{+} \times \mathbb{R} .
$$

Then there exists at least one mild solution $u \in C\left([-\tau,+\infty) ; H_{0}^{1}(\Omega)\right)$ with $\frac{\partial u}{\partial t} \in$ $C\left([-\tau,+\infty) ; L^{2}(\Omega)\right)$ of problem (4.1).

Proof. We will prove only the existence of a local mild solution, the extendability of this to a global one follows from hypothesis $\left(\mathrm{h}_{1}\right)$ combined with Burlică and Roşu [4, Lemma 4.3, p. 2452]. Problem (4.1) can be rewritten as a first-order system of partial differential equations of the form

$$
\begin{cases}\frac{\partial u}{\partial t}(t, x)=v(t, x) & \text { in } Q_{+}, \\ \frac{\partial v}{\partial t}(t, x)=\Delta u(t, x)+h\left(t, \int_{-\tau}^{0} u(t+s, x) d s\right) & \text { in } Q_{+}, \\ u(t, x)=0 & \text { on } \Sigma_{+}, \\ u(t, x)=\psi_{1}(t)(x) & \text { in } Q_{\tau}, \\ v(t, x)=\psi_{2}(t)(x) & \text { in } Q_{\tau} .\end{cases}
$$


The product space $X$, defined by (4.2), endowed with the natural inner product

$$
\left\langle\left(\begin{array}{c}
u \\
v
\end{array}\right),\left(\begin{array}{c}
\widetilde{u} \\
\widetilde{v}
\end{array}\right)\right\rangle=\int_{\Omega} \nabla u(x) \cdot \nabla \widetilde{u}(x) d x+\int_{\Omega} v(x) \widetilde{v}(x) d x,
$$

for each $\left(\begin{array}{l}u \\ v\end{array}\right),\left(\begin{array}{c}\widetilde{u} \\ v\end{array}\right) \in X$, is a real Hilbert space. Clearly, (4.3) can be rewritten as an abstract evolution equation subjected to nonlocal initial conditions of the form (3.2) in the space $X$, where $A, F$ and $\varphi$ are defined as follows.

First, let us define the linear operator $A: D(A) \subseteq X \rightarrow X$ by

$$
\begin{gathered}
D(A)=\left(\begin{array}{c}
H_{0}^{1}(\Omega) \cap H^{2}(\Omega) \\
\times \\
H_{0}^{1}(\Omega)
\end{array}\right), \\
A\left(\begin{array}{c}
u \\
v
\end{array}\right):=\left(\begin{array}{c}
v \\
\Delta u
\end{array}\right), \quad \text { for each }\left(\begin{array}{c}
u \\
v
\end{array}\right) \in D(A) .
\end{gathered}
$$

By Vrabie [15, Theorem 4.6.2, p. 93], we know that the linear operator $A$, previously defined, is the infinitesimal generator of a $C_{0}$-group of unitary operators $\{G(t): X \rightarrow X ; t \in \mathbb{R}\}$. Now, let us define $F: \mathbb{R}_{+} \times X \rightarrow X$ by

$$
F\left(t,\left(\begin{array}{c}
u \\
v
\end{array}\right)\right)(x):=\left(\begin{array}{c}
0 \\
h(t, u(x))
\end{array}\right),
$$

for each $t \in \mathbb{R}_{+}$, each $\left(\begin{array}{c}u \\ v\end{array}\right) \in X$ and for almost every $x \in \Omega$. So, problem (4.3) rewrites in the space $X$ as

$$
\begin{cases}\left(\begin{array}{l}
u \\
v
\end{array}\right)^{\prime}(t)=A\left(\begin{array}{c}
u \\
v
\end{array}\right)(t)+F\left(t, \int_{-\tau}^{0}\left(\begin{array}{c}
u(t+s) \\
v(t+s)
\end{array}\right) d s\right) & \text { if } t \in \mathbb{R}_{+}, \\
\left(\begin{array}{c}
u(t) \\
v(t)
\end{array}\right)=\left(\begin{array}{c}
\psi_{1}(t) \\
\psi_{2}(t)
\end{array}\right) & \text { if } t \in[-\tau, 0] .\end{cases}
$$

Thus, as we have already noticed in Remark 4.2, (4.3) rewrites under the form (3.2), where $A, F$ are defined as above and $\varphi:=\left(\begin{array}{l}\psi_{1} \\ \psi_{2}\end{array}\right)$.

From Lemma 4.1, we conclude that $F$ is continuous and compact and thus satisfies the hypotheses of Theorem 3.2, from where the local existence part of the conclusion follows. As the global existence part is standard, this completes the proof.

REMARK 4.4. If we are merely interested in obtaining local solutions, linear growth condition $\left(\mathrm{h}_{1}\right)$ on the function $h$ can be relaxed according to the dimension of $\mathbb{R}^{d}$. Namely, if $d=1$ and $h$ is continuous or if $d=2$ and $h$ satisfies any polynomial growth condition, by means of Theorem 1.6, we can prove the local existence part in Theorem 4.3. If $d \geq 3$ and $h$ satisfies 
$\left(\mathrm{h}_{2}\right) h$ is continuous and there exist $\widetilde{\ell}>0, \widetilde{m} \geq 0$ and $\alpha \in(0, d /(d-2))$ such that

$$
|h(t, y)| \leq \widetilde{\ell}|y|^{\alpha}+\widetilde{m}, \quad \text { for all }(t, y) \in \mathbb{R}_{+} \times \mathbb{R},
$$

also by Theorem 1.6, we can get the local existence.

\section{An application to a problem in mechanics}

Let $\Omega$ be a nonempty bounded and open subset in $\mathbb{R}^{d}, d \geq 1$, with $C^{1}$ boundary $\Sigma$. The controlled movement of a continuous medium, having the domain $\Omega$, is described by the following pseudoparabolic semilinear partial differential equation:

$$
\begin{cases}\frac{\partial u}{\partial t}=\alpha \Delta \frac{\partial u}{\partial t}+\beta \Delta u+h\left(t, x, \int_{-\tau}^{0} u(t+s, x) d s\right), & (t, x) \in Q_{+} \\ u=0, & (t, x) \in \Sigma_{+} \\ u(t, x)=\varphi(t)(x), & (t, x) \in Q_{\tau}\end{cases}
$$

where $\alpha>0, \beta>0, h: \mathbb{R}_{+} \times \bar{\Omega} \times \mathbb{R}$ is continuous and $\varphi \in C\left([-\tau, 0] ; L^{2}(\Omega)\right)$ satisfies $\varphi(t) \in H^{2}(\Omega) \cap H_{0}^{1}(\Omega)$ for each $t \in[-\tau, 0]$ and $Q_{+}, Q_{\tau}, \Sigma_{+}$are as in Section 4 .

In the case $\tau=0$ and $h$ depending only on the first two arguments, equations of form (5.1) describe the evolution of various phenomena such as the flow of fluid through fissured rocks, heat conduction involving two temperatures, shear in second-order fluids and are of interest in soil mechanics. For details see Brill [3] and the references therein. In its general form, problem (5.1) represents the closed loop system associated to a control problem governed by a delay differential equation of the form mentioned above, $h$ being the synthesis operator.

THEOREM 5.1. In the general hypotheses above, let us assume that there exist $c>0$ and $d \geq 0$ such that

$$
|h(t, x, u)| \leq c|u|+d, \quad \text { for all }(t, x, u) \in \mathbb{R}_{+} \times \bar{\Omega} \times \mathbb{R} .
$$

Then problem (5.1) has at least one solution $u \in C^{1}\left(\mathbb{R}_{+} ; H_{0}^{1}(\Omega) \cap H^{2}(\Omega)\right)$.

Proof. As before, we confine ourselves only to the proof of the local existence. Let $A: D(A) \subseteq L^{2}(\Omega) \rightarrow L^{2}(\Omega)$ be defined by

$$
\left\{\begin{array}{l}
D(A)=H_{0}^{1}(\Omega) \cap H^{2}(\Omega), \\
A u=\Delta u \text { for each } u \in D(A) .
\end{array}\right.
$$


Problem (5.1) can be rewritten as an implicit differential equation in the space $L^{2}(\Omega)$, i.e.

$$
\left\{\begin{array}{l}
{[(I-\alpha A) u]^{\prime}=\beta A u+\widetilde{h}\left(t, \int_{-\tau}^{0} u(t+s) d s\right),} \\
u(t)=\varphi(t)
\end{array} \quad \text { if } t \in[-\tau, 0],\right.
$$

where $\widetilde{h}: \mathbb{R}_{+} \times L^{2}(\Omega) \rightarrow L^{2}(\Omega)$ is defined by

$$
\widetilde{h}(t, v)(x):=h(t, x, v(x)), \quad \text { for each } v \in L^{2}(\Omega) \text { and for a.e. } x \in \Omega \text {. }
$$

Let us denote $(I-\alpha A) u$ by $v$ and let us observe that

$$
\beta A(I-\alpha A)^{-1}=\frac{\beta}{\alpha}[I-(I-\alpha A)](I-\alpha A)^{-1}=\frac{\beta}{\alpha}(I-\alpha A)^{-1}-\frac{\beta}{\alpha} I .
$$

So, problem (5.2) can be reformulated as

$$
\begin{cases}v^{\prime}(t)=f\left(t, v_{t}\right)+g\left(t, v_{t}\right) & \text { if } t \in \mathbb{R}_{+}, \\ v(t)=\psi(t) & \text { if } t \in[-\tau, 0],\end{cases}
$$

where the function $f: \mathbb{R}_{+} \times C\left([-\tau, 0] ; L^{2}(\Omega)\right) \rightarrow L^{2}(\Omega)$ is defined by

$$
f(t, w):=\widetilde{h}\left(t,(I-\alpha A)^{-1} \int_{-\tau}^{0} w(s) d s\right),
$$

for each $t \in \mathbb{R}_{+}$and each $w \in C\left([-\tau, 0] ; L^{2}(\Omega)\right)$. Moreover, the function $g: \mathbb{R}_{+} \times$ $C\left([-\tau, 0] ; L^{2}(\Omega)\right) \rightarrow L^{2}(\Omega)$ is given by

$$
g(t, w):=\omega(I-\alpha A)^{-1} w(0)-\omega w(0),
$$

for each $t \in \mathbb{R}_{+}$and each $w \in C\left([-\tau, 0] ; L^{2}(\Omega)\right)$, where $\omega=\beta \alpha^{-1}>0$ and

$$
\psi(t)(x):=(I-\alpha A) \varphi(t)(x),
$$

for each $t \in[-\tau, 0]$ and for almost every $x \in \Omega$. Taking into account that $(I-\alpha A)^{-1}$ is continuous from $L^{2}(\Omega)$ to $H^{2}(\Omega) \cap H_{0}^{1}(\Omega)$ and, by Theorem 1.6, the latter is compactly imbedded in $C(\bar{\Omega})$, from Lemma 1.3 , it follows that the function $f$ is compact. Furthermore, as $\omega(I-\alpha A)^{-1}-\omega I$ is Lipschitz continuous it follows that $g$ is continuous on $\mathbb{R}_{+} \times C\left([-\tau, 0] ; L^{2}(\Omega)\right)$ and Lipschitz with respect to its second variable. So, we are in the hypotheses of Theorem 2.1, from where it follows that problem (5.3) has at least one local classical solution $v:[-\tau, T] \rightarrow L^{2}(\Omega)$. Then $u=(A-\alpha I)^{-1} v$ is a local solution for problem (5.1), satisfying the additional regularity property: $u \in C^{1}\left([0, T] ; H^{2}(\Omega) \cap H_{0}^{1}(\Omega)\right)$. Since by $\left(\mathrm{h}_{1}\right)$ it follows that $f$ has linear growth while $g$ is linear continuous, we conclude that this local solution can be continued up to a global one and this completes the proof of Theorem 5.1. 


\section{REFERENCES}

[1] R.A. Adams, Sobolev spaces, Academic Press, Boston, 1978.

[2] R.I. Becker, Periodic solutions of semilinear equations of evolution of compact type, J. Math. Anal. Appl. 82 (1981), 33-48.

[3] H. BRILl, A semilinear Sobolev evolution equation in a Banach space, J. Differential Equations 24 (1977), 412-425.

[4] M.D. Burliç And D. RoşU, A class of nonlinear delay evolution equations with nonlocal initial conditions, Proc. Amer. Math. Soc. 142 (2014), 2445-2458.

[5] O. CÂrJă, M. Necula and I.I. Vrabie, Viability, Invariance and Applications, HorthHolland Mathematics Studies 207, Elsevier, Amsterdam, 2007.

[6] R.D. Driver, Ordinary and delay differential equations, Appl. Math. Sci. 20, Springer Verlag, New York, 1977.

[7] N. Dunford And J.T. Schwartz, Linear Operators Part I: General Theory, Interscience Publishers, Inc. New York, 1958.

[8] M. Frigon and D. O'Reagan, Existence results for initial value problems in Banach spaces, Differ. Equ. Dyn. Syst. 2 (1994), 41-48.

[9] A. Halanay, Differential Equations, Stability, Oscillations, Time Lags, Academic Press, New York and London, 1966.

[10] J. Hale, Functional differential equations, Applied Mathematical Sciences 3, Springer Verlag, 1971.

[11] M. A. KrasnoseL'SKIII, Two remarks on the method of successive approximations, Uspehi Mat. Nauk 10 (1955), 123-127 (Russian).

[12] E. Mitidieri And I.I. VRABie, Existence for nonlinear functional differential equations, Hiroshima Math. J. 17 (1987), 627-649.

[13] _ A class of strongly nonlinear functional differential equations, Ann. Mat. Pura Appl. (4) CLI (1988), 125-147.

[14] J. Schauder, Der Fixpunktsatz in Funktionalräumen, Studia Math. 2 (1930), 171-180.

[15] I.I. Vrabie, $C_{0}$-semigroups and Applications, North-Holland, Amsterdam, 2003.

IOAN I. VRABIE

"Al. I. Cuza" University

Faculty of Mathematics

Iaşi 700506, ROMANIA

and

"Octav Mayer" Mathematics Institute

of the Romanian Academy

Iaşi 700505, ROMANIA

E-mail address: ivrabie@uaic.ro 